\section{Aspectos relacionados à escolha do tipo de parto: um estudo comparativo entre uma maternidade pública e outra privada, em São Luís, Maranhão, Brasil}

\author{
Aspects related to choice of type of delivery: \\ a comparative study of two maternity hospitals \\ in São Luís, State of Maranhão, Brazil
}

\author{
1 Programa de Pós-graduação \\ em Saúde Materno-Infantil, \\ Universidade Federal do \\ Maranhão, São Luís, Brasil. \\ 2 Departamento de Medicina, \\ Universidade Federal do \\ Maranhão, São Luís, Brasil. \\ Correspondência \\ N. R. Mandarino \\ Programa de Pós-graduação \\ em Saúde Materno-Infantil. \\ Universidade Federal do \\ Maranhão. \\ Rua Santa Luzia 27, QD 17, \\ São Luís, MA \\ 65067-460, Brasil. \\ nmandarino@bol.com.br
}

\section{Abstract}

This study aimed to analyze aspects related to choice of type of delivery in two maternity hospitals, one public and the other private, in São Luís, Maranhão State, Brazil. This cross-sectional study compared 163 primiparous women in a public maternity hospital and 89 in a private hospital, with mean ages of $21.63 \pm 5.24$ and $28.8 \pm 5.41$ years, respectively. In the public hospital, $79.1 \%$ of the women reported preferring vaginal deliveries, while in the private hospital $67.4 \%$ of the women preferred cesareans $(p<0.0001)$. Cesareans were performed in $46 \%$ of the women in the public maternity hospital and $97.8 \%$ of those in the private hospital $(p<$ 0.0001). Patient satisfaction was high for both modes of delivery, but the desire to repeat the same mode was reported more frequently by women with vaginal deliveries (71.6\% vs. 41.3\% in the public maternity hospital and $100 \% \mathrm{vs}$. $65.5 \%$ in the private). In the public maternity hospital, the cesarean subgroup included more white and higher-income women. The cesarean rate was thus high in both maternity hospitals and was significantly higher in the private hospital; the study also showed a preference for vaginal delivery in the public hospital and cesareans in the private.

Obstetric Delivery; Maternity Hospitals; Comparative Study
Natália Ribeiro Mandarino 1

Maria Bethânia da Costa Chein 1

Francisco das Chagas Monteiro Júnior ${ }^{2}$

Luciane Maria Oliveira Brito 1

Zeni Carvalho Lamy 1

Vinícius José da Silva Nina 2

Elba Gomide Mochel 1

José Albuquerque de Figueiredo Neto 1

\section{Introdução}

A escolha do tipo de parto, vaginal (normal) ou cirúrgico (cesárea ou cesariana), é assunto complexo e polêmico. A cesariana, outrora considerada um procedimento de exceção, indicada em situações de risco de vida para a gestante e/ou feto, é na atualidade um procedimento cirúrgico na maioria das vezes programado, sem a identificação médica de nenhum risco definido, cuja escolha é freqüentemente atribuída à gestante $1,2,3,4,5,6,7,8$.

A Organização Mundial da Saúde (OMS) preconiza como ideal uma taxa de cesarianas entre $10 \%$ e $15 \%$. No entanto, o que se tem observado são taxas universais em geral superiores, até em países considerados desenvolvidos 9. À semelhança de outros países em desenvolvimento, o Brasil apresenta taxas de cesarianas superiores àquelas preconizadas pela OMS, superando os $35 \%$ em geral e ultrapassando os $70 \%$, quando se considera apenas o serviço privado 1,5,10,11,12.

Entre as Unidades Federativas brasileiras não existe nenhuma que apresente valores dentro dos parâmetros desejáveis pela OMS. O Amapá, localizado no extremo norte do país, e São Paulo, na Região Sudeste (socioeconomicamente mais desenvolvida) apresentam, respectivamente, a menor $(23,42 \%)$ e a maior (53\%) taxa proporcional de cesarianas realizadas pelo Sistema Único de Saúde (SUS) (Departamento de Informática do SUS. Indicadores e dados básicos, 2006. http:// 
tabnet.datasus.gov.br/cgi/tabcgi.exe?idb2006/ f08.def., acessado em 20/Mar/2008). Tais cifras têm colocado o Brasil em posição de destaque no panorama mundial 12,13.

As cesáreas acarretam aumento da morbimortalidade materna e neonatal, destacando-se a infecção puerperal e a prematuridade. Também se associam com um retardo na recuperação puerperal, maior tempo de internação, maior tempo de assistência por profissionais de saúde, durante a internação mais prolongada, maior uso de medicamentos, início tardio da amamentação e, por fim, elevação de gastos para o sistema de saúde. Parece que o incremento das cesarianas não se deve apenas a questões médicas, sendo influenciado por diversos outros fatores relacionados à gestante, como as desigualdades sócio-econômicas, a situação geográfica, a faixa etária e a etnia 5,12,13,14,15.

Os médicos, muitas vezes apontados como promotores de uma cultura intervencionista por conveniência, alegam que muitas vezes a realização da cesariana é justificada também pelo desejo das gestantes, principalmente as de classes sociais mais favorecidas, que preferem esta via para preservar a anatomia da genitália externa, para não sentir a dor do trabalho de parto e, entre aquelas com desejo de definir a prole, para aproveitar a ocasião e realizar a laqueadura tubária (esterilização cirúrgica) 5,11,12,13,15,16,17.

A “medicalização" do parto, ou seja, a mudança de assistência baseada na plausível segurança dos procedimentos médicos intervencionistas, transformou o parto normal em um parto passível de intervenções, ou seja, de risco. Assim, o parto normal, na atualidade, passou a significar parto vaginal dirigido ou orientado, em ambiente hospitalar. A tecnologia médica se apresenta, nesse sentido, como uma resposta necessária para o controle desse risco, justificando a legitimização social do parto cesáreo como um procedimento seguro, indolor, moderno e ideal para qualquer grávida, adaptando-o para que se insira na orientação geral da medicina ocidental de beneficência ("poupar a mulher da dor") 18.

Sob a égide do discurso feminista acerca do direito da mulher à escolha do parto, a prática obstétrica apropria-se deste discurso para justificar o pedido da gestante para a realização da cesariana ("cesárea a pedido"). Porém, a aparente "liberdade de escolha" outorgada à mulher é, muitas vezes, acompanhada da falta de informações adequadas sobre os riscos envolvidos nos procedimentos relacionados ao parto e ao nascimento $11,19,20$.
É evidente que as taxas crescentes de cesarianas sem indicação médica, tanto em países desenvolvidos como nos em desenvolvimento, têm motivado diversas pesquisas e debates sobre a legalidade do procedimento cirúrgico sem uma necessidade médica identificada. Nesse contexto, a Federação Internacional das Sociedades de Ginecologia e Obstetrícia (FIGO), que congrega todos os toco-ginecologistas brasileiros, por intermédio da Associação Brasileira das Sociedades de Ginecologia e Obstetrícia (FEBRASGO), por meio de seu Comitê de Aspectos Éticos da Reprodução Humana, apontou que não é ética a prática de cesariana sem uma indicação médica formal 19 .

Contrariamente a essa posição, o Comitê de Ética do Colégio Americano de Ginecologistas e Obstetras (ACOG) reafirma que se deve respeitar o direito da mulher ("autonomia do sujeito") a decidir a via de parto, em pré-natal de baixo risco, estando a gravidez entre 39 e 40 semanas, desde que a mesma esteja informada e devidamente esclarecida sobre os riscos e benefícios do procedimento cirúrgico e assine um termo de consentimento livre e esclarecido, posição esta que tem suscitado debates e questionamentos éticos 6,16,21.

A compreensão da diversidade de fatores que interferem na assistência ao parto, culminando nessas taxas elevadas de cesarianas, ainda carece de consenso na proposição de soluções para o controle dessas taxas.

No Município de São Luís, Maranhão, existem 13 hospitais que realizam assistência ao parto. No ano de 2006, foram realizados mensalmente, em média, $1.435,5$ partos no serviço público (inclusive o conveniado ao SUS). Do total de 17.226 partos realizados no referido ano, 3.703 foram cesarianas $(27,38 \%) 22$. Dados do serviço privado não estão disponíveis oficialmente. Além disso, outras informações relevantes, como a preferência das gestantes pela via de parto e os motivos alegados, não são de todo conhecidas. Tampouco se sabe se essa preferência está sendo correspondida, bem como se as parturientes estão satisfeitas com o tipo de parto realizado. É possível que fatores não-clínicos, como nível sócio-econômico, informação inadequada e conveniência do médico, realmente influenciem na escolha da via de parto, como sugerem alguns autores 11,12,13,16.

Assim, a presente pesquisa se propõe a conhecer os desejos das parturientes relacionados a cada via de parto, bem como verificar e comparar a freqüência de cesarianas, procurando-se identificar as suas indicações e outras variáveis determinantes da via de parto em nosso meio. 


\section{Materiais e métodos}

Realizou-se um estudo transversal em duas unidades hospitalares, uma pública e outra privada, ambas consideradas de referência para atendimento obstétrico e perinatal no estado, nos meses de fevereiro e março, na primeira, e abril e maio de 2007, na segunda.

A maternidade pública localiza-se no centro da capital e atua como hospital de ensino, pesquisa e extensão, destinando $100 \%$ de seus leitos aos usuários do SUS e oferecendo atendimento ambulatorial e de urgência e emergência. A maternidade privada localiza-se também na capital, em bairro próximo ao centro da cidade, prestando também atendimento ambulatorial e de urgência e emergência, não destinando nenhum leito para usuários do SUS.

Participaram do estudo todas as primíparas internadas consecutivamente nos referidos períodos em trabalho de parto, sendo incluídas 163 mulheres na maternidade pública e 89 na privada. Para comparação da proporção de cesarianas entre as duas unidades, estimou-se uma taxa de $60 \%$ para a unidade pública e de $90 \%$ para a privada, sendo calculado um número mínimo de 49 casos para cada unidade, adotando-se uma probabilidade de erro tipo I de $5 \%$ e poder de $80 \%$. A não inclusão de multíparas neste trabalho visou a evitar-se uma tendenciosidade na escolha do tipo de parto seguinte. Como comenta a socióloga americana Hopkins 11, que realizou pesquisa em maternidades brasileiras, a via do primeiro parto é crucial como determinante da escolha do tipo do(s) futuro(s) parto(s), pois é senso comum, tanto entre os médicos como entre as mulheres, que "uma vez cesárea, sempre cesárea".

Foram considerados critérios de exclusão a apresentação de qualquer condição clínica que por si só representasse uma indicação para o parto cesáreo (por exemplo: endocrinopatias, cardiopatias) e a incapacidade de responder ao questionário sem a participação de outra pessoa, não tendo ocorrido, no entanto, nenhum caso de exclusão.

Na fase do pré-parto, após a explicação dos objetivos da pesquisa e anuência textual (assinatura do Termo de Consentimento Livre e Esclarecido-TCLE), todas participaram da primeira parte da entrevista, respondendo a um questionário com perguntas fechadas e abertas sobre qual a via de parto de sua preferência.

Decorridas 24 a 36 horas após o parto, todas foram submetidas à segunda parte do mesmo questionário para se obterem outras informações: características sócio-demográficas (idade, cor, escolaridade, profissão, situação conjugal, renda), assistência pré-natal, idade da menarca e da primeira relação sexual, tipo do parto realizado (vaginal ou cesariano), satisfação com o tipo de parto realizado e, se repetiria o mesmo tipo de parto, e em caso de cesariana, se a mesma teve uma indicação médica (desproporção céfalopélvica, sofrimento fetal, apresentação pélvica, gemelaridade e outras), considerando-se primariamente a anotação constante no prontuário, ou realizou-se "a pedido", informação obtida baseando-se na entrevista com a gestante, na falta de anotação médica de uma indicação.

A idade foi categorizada por faixas: menor que 20 anos (adolescentes), de 20 a 34 anos (adultas jovens) e maior ou igual a 35 (adultas).

Considerou-se a assistência pré-natal completa quando a gestante realizou mais de seis consultas.

As duas populações, da maternidade pública e da privada, foram comparadas quanto às características sócio-demográficas, preferência das parturientes em relação ao tipo de parto, fre qüência de cesarianas, motivos para a realização das mesmas e satisfação das pacientes com o tipo de parto realizado. Em cada uma, procurouse identificar as variáveis associadas ao tipo de parto realizado.

Os dados obtidos foram expressos por meio de tabelas. As variáveis qualitativas foram representadas por freqüência relativa (\%) e as variáveis contínuas expressas como média e desviopadrão. Para análise estatística foi utilizado o programa Epi Info versão 3.3.2 (Centers for Disease Control and Prevention, Atlanta, Estados Unidos), adotando-se como valor significativo um $\mathrm{p}<0,05$. O teste qui-quadrado foi adotado para cálculo de significância na comparação univariada de proporções.

Esta pesquisa foi aprovada pelo Comitê de Ética em Pesquisa do Hospital Universitário da Universidade Federal do Maranhão sob o no. 33104-0063/2007 e está de acordo com os princípios éticos contidos na Declaração de Helsinki.

\section{Resultados}

A média de idade das duas populações, da maternidade pública e da privada, foi de 21,63 $\pm 5,24 \mathrm{e}$ $28,8 \pm 5,41$ anos, respectivamente. Como pode ser observado na Tabela 1, na população atendida na maternidade pública havia um predomínio de mulheres pardas e com níveis de escolaridade e renda mais baixos.

Quanto à via de parto, 79,1\% das gestantes da maternidade pública afirmaram preferir o parto vaginal, enquanto que na maternidade privada foi o parto cesáreo que deteve a preferência da maioria das mulheres $(67,4 \%)$, com diferença es- 
Distribuição das mulheres de acordo com as características sociodemográficas. São Luís, Maranhão, Brasil, 2007.

\begin{tabular}{|c|c|c|c|c|c|}
\hline \multirow[t]{3}{*}{ Variáveis } & \multicolumn{4}{|c|}{ Maternidade } & \multirow[t]{3}{*}{ Valor de $p$} \\
\hline & \multicolumn{2}{|c|}{ Pública (n = 163) } & \multicolumn{2}{|c|}{ Privada $(n=89)$} & \\
\hline & $f$ & $\%$ & $f$ & $\%$ & \\
\hline Idade (anos) & & & & & $<0,0001$ \\
\hline$\leq 19$ & 66 & 40,5 & 2 & 2,2 & \\
\hline $20-34$ & 94 & 57,7 & 76 & 85,4 & \\
\hline$\geq 35$ & 3 & 1,8 & 11 & 12,4 & \\
\hline Cor & & & & & $<0,0001$ \\
\hline Branca & 35 & 21,5 & 53 & 59,6 & \\
\hline Parda & 100 & 61,3 & 34 & 38,2 & \\
\hline Negra & 28 & 17,2 & 2 & 2,2 & \\
\hline Escolaridade & & & & & $<0,0001$ \\
\hline Ensino Fundamental & 55 & 35,5 & - & - & \\
\hline Ensino Médio & 91 & 55,8 & 39 & 43,8 & \\
\hline 3으 Grau & 14 & 8,6 & 50 & 56,2 & \\
\hline Ignorada & 3 & 0,1 & - & - & \\
\hline Estado civil & & & & & $<0,1486$ \\
\hline Solteira/Viúva & 60 & 36,8 & 24 & 27,0 & \\
\hline Casada/Vida marital & 103 & 63,2 & 65 & 73,0 & \\
\hline Renda familiar (salários mínimos) & & & & & $<0,0001$ \\
\hline$<1$ & 28 & 17,2 & - & - & \\
\hline $1<3$ & 91 & 55,8 & - & - & \\
\hline$\geq 3$ & 18 & 11,0 & 84 & 94,4 & \\
\hline Outros & 26 & 16,0 & 5 & 5,6 & \\
\hline Ocupação & & & & & $<0,0001$ \\
\hline Desempregada & 53 & 32,5 & - & - & \\
\hline Dona-de-casa & 48 & 29,4 & 9 & 10,1 & \\
\hline Estudante & 23 & 14,1 & 18 & 20,2 & \\
\hline Profissionais liberais & - & - & 14 & 15,7 & \\
\hline Outros & 39 & 24,0 & 59 & 54,0 & \\
\hline
\end{tabular}

tatisticamente significativa $(\mathrm{p}<0,0001)$. Nas duas maternidades, o principal motivo alegado para a preferência pelo parto vaginal foi o fato de a recuperação ser mais rápida; para as mulheres que manifestaram preferência pelo parto cesáreo, o motivo mais freqüentemente apontado foi o medo de sentir dor (Tabela 2).

Tanto na maternidade pública como na privada, a maioria das gestantes referiu ter recebido assistência pré-natal (96,9\% e 98,9\%, respectivamente), sem diferença estatisticamente significante ( $p=0,59)$. Essa assistência foi considerada completa em $57 \%$ das gestantes da maternidade pública e em $97,8 \%$ das da privada. Na maternidade pública, 3 mulheres (1,9\%) não souberam referir o número de consultas prénatais realizadas.

O parto cesáreo foi realizado em 75 mulheres (46\%) da maternidade pública e em 87 (97,8\%) da maternidade privada ( $\mathrm{p}<0,0001)$. Encontrou-se indicação clínica para a realização das cesarianas em 94,7\% das gestantes da maternidade pública, enquanto que na privada, em apenas $63,2 \%$ dos casos havia registro no prontuário de uma indicação clínica $(\mathrm{p}<0,0001)$. Para as demais gestantes da maternidade privada que realizaram cesariana $(36,8 \%)$ não havia documentação de indicação da mesma, ficando esclarecido que foi "a pedido" baseando-se na informação da parturiente durante a entrevista no pós-parto (Tabela 3).

Em relação às indicações clínicas da cesariana, encontrou-se uma maior freqüência de desproporção céfalo-pélvica em ambos os serviços (37,3\% e $28,7 \%$, respectivamente). A Tabela 4 relaciona a freqüência das demais indicações.

As taxas de concordância entre a preferência da gestante e o tipo de parto realizado na ma- 
Distribuição das mulheres de acordo com a preferência pela via de parto. São Luís, Maranhão, Brasil, 2007.

\begin{tabular}{|c|c|c|c|c|}
\hline \multirow[t]{3}{*}{ Variáveis } & \multicolumn{4}{|c|}{ Maternidade } \\
\hline & \multicolumn{2}{|c|}{ Pública ( $n=163$ ) } & \multicolumn{2}{|c|}{ Privada $(\mathrm{n}=89)$} \\
\hline & $f$ & $\%$ & $f$ & $\%$ \\
\hline \multicolumn{5}{|l|}{ Preferência da gestante * } \\
\hline Parto vaginal & 129 & 79,1 & 29 & 32,6 \\
\hline Parto cesariana & 34 & 20,9 & 60 & 67,4 \\
\hline \multicolumn{5}{|l|}{ Motivo da preferência } \\
\hline \multicolumn{5}{|l|}{ Vaginal } \\
\hline Recuperação é mais rápida & 95 & 73,6 & 26 & 89,7 \\
\hline É natural & 26 & 20,2 & - & - \\
\hline Habitual na família & 5 & 3,9 & - & - \\
\hline Medo da anestesia & - & - & 2 & 6,9 \\
\hline Outros & 3 & 2,3 & 1 & 3,4 \\
\hline Total & 129 & 100,0 & 29 & 100,0 \\
\hline \multicolumn{5}{|l|}{ Cesariana } \\
\hline Medo de sentir dor & 28 & 82,4 & 54 & 90,0 \\
\hline Orientação/Indicação de amigos & 1 & 2,9 & 3 & 5,0 \\
\hline Evitar deformações vaginais & 2 & 5,9 & 2 & 3,3 \\
\hline Recomendação do médico & 3 & 8,8 & 1 & 1,7 \\
\hline Total & 34 & 100,0 & 60 & 100,0 \\
\hline
\end{tabular}

$\star p<0,001$

Tabela 3

Distribuição das mulheres de acordo com o tipo de parto realizado e motivo da cesariana. São Luís, Maranhão, Brasil, 2007.

\begin{tabular}{|c|c|c|c|c|c|}
\hline \multirow[t]{3}{*}{ Variáveis } & \multicolumn{4}{|c|}{ Maternidade } & \multirow[t]{3}{*}{ Valor de $p$} \\
\hline & \multicolumn{2}{|c|}{ Pública ( $n=163$ ) } & \multicolumn{2}{|c|}{ Privada $(n=89)$} & \\
\hline & $f$ & $\%$ & $f$ & $\%$ & \\
\hline Tipo de parto & & & & & $<0,0001$ \\
\hline Vaginal & 88 & 54,0 & 2 & 2,2 & \\
\hline Cesariano & 75 & 46,0 & 87 & 97,8 & \\
\hline Motivo da cesariana & & & & & $<0,0001$ \\
\hline Indicação clínica & 71 & 94,7 & 55 & 63,2 & \\
\hline "A pedido" & 4 & 5,3 & 32 & 36,8 & \\
\hline
\end{tabular}

ternidade pública foram de 55,8\% para o parto vaginal e $38,2 \%$ para o parto cesáreo; na maternidade privada foram de 6,9\% para o parto vaginal e $100 \%$ para o parto cesáreo.

Nas duas maternidades, o nível de satisfação com ambas as vias de parto foi elevado: $93,8 \%$ com a via vaginal e $90,7 \%$ com a via abdominal na maternidade pública e $100 \%$ com ambas as vias na privada. Na maternidade pública, $71,6 \%$ das mulheres submetidas ao parto vaginal responderam que repetiriam esta via de parto, con- tra $41,3 \%$ das que se submeteram à cesariana; os porcentuais para a maternidade privada foram de, respectivamente, $100 \%$ e $65,5 \%$.

Comparando o grupo do parto vaginal com o da cesariana na maternidade pública, verificouse não haver diferença significante em relação às variáveis: idade, estado civil, escolaridade, assistência pré-natal e se o pré-natal foi completo ou não. Em relação à cor, havia mais brancas no grupo do parto abdominal e mais pardas no grupo do parto vaginal $(p=0,0163)$. Verificou-se 
ainda uma maior proporção de renda acima de três salários mínimos no grupo cesariano $(\mathrm{p}=$ 0,0001) (Tabela 5).

\section{Discussão}

As taxas de cesarianas registradas no Brasil diferem bastante conforme a instituição considerada, se pública ou privada. Assim, nesta pesquisa, foram escolhidas para análise duas maternidades, uma pública e outra privada, consideradas de referência na cidade. Tratando-se de populações bem distintas, as mulheres atendidas na maternidade privada apresentavam, como era de se esperar, níveis mais elevados de escolaridade e renda, predominando a cor branca.

Quanto à via de parto, quase $80 \%$ das parturientes da maternidade pública manifestaram preferência pelo parto vaginal, achado concor-

Tabela 4

Distribuição das mulheres de acordo com as indicações do parto cesáreo. São Luís, Maranhão, Brasil, 2007.

\begin{tabular}{|c|c|c|c|c|}
\hline \multirow[t]{3}{*}{ Variáveis } & \multicolumn{4}{|c|}{ Maternidade } \\
\hline & \multicolumn{2}{|c|}{ Pública (n = 71) } & \multicolumn{2}{|c|}{ Privada $(n=55)$} \\
\hline & $f$ & $\%$ & $f$ & $\%$ \\
\hline Desproporção céfalo-pélvica & 31 & 43,7 & 25 & 28,7 \\
\hline DHEG & 21 & 29,6 & 12 & 13,8 \\
\hline Sofrimento fetal & 18 & 25,3 & 15 & 17,2 \\
\hline Gemelaridade & 1 & 1,4 & 3 & 3,4 \\
\hline
\end{tabular}

DHEG: doença hipertensiva específica da gravidez.

Tabela 5

Distribuição das características das parturientes da maternidade pública de acordo com a via de parto. São Luís, Maranhão, Brasil, 2007.

\begin{tabular}{|c|c|c|c|c|c|}
\hline \multirow[t]{3}{*}{ Variáveis } & \multicolumn{4}{|c|}{ Tipo de parto } & \multirow[t]{3}{*}{ Valor de $\mathrm{p}$} \\
\hline & \multicolumn{2}{|c|}{ Vaginal $(n=88)$} & \multicolumn{2}{|c|}{ Cesáreo $(n=75)$} & \\
\hline & $f$ & $\%$ & $f$ & $\%$ & \\
\hline Idade (anos) & & & & & 0,506 \\
\hline$\leq 19$ & 38 & 43,2 & 28 & 37,3 & \\
\hline $20-34$ & 48 & 54,5 & 46 & 61,3 & \\
\hline$\geq 35$ & 2 & 2,3 & 1 & 1,4 & \\
\hline Cor & & & & & 0,0163 \\
\hline Branca & 12 & 13,6 & 23 & 30,7 & \\
\hline Parda & 62 & 70,5 & 38 & 50,7 & \\
\hline Negra & 14 & 15,9 & 14 & 18,7 & \\
\hline Escolaridade & & & & & 0,0714 \\
\hline Ensino Fundamental & 36 & 41,0 & 20 & 27,7 & \\
\hline Ensino Médio & 47 & 53,4 & 45 & 60,0 & \\
\hline 3o Grau & 5 & 5,6 & 10 & 13,3 & \\
\hline Estado civil & & & & & 0,9721 \\
\hline Solteira/Viúva & 33 & 37,5 & 27 & 36,0 & \\
\hline Casada/Vida marital & 55 & 62,5 & 48 & 64,0 & \\
\hline Renda familiar (salários mínimos) & & & & & $<0,0001$ \\
\hline$<1$ & 15 & 17,0 & 13 & 17,3 & \\
\hline $1<3$ & 51 & 58,0 & 40 & 53,3 & \\
\hline$\geq 3$ & 5 & 5,3 & 22 & 29,2 & \\
\hline Outros & 17 & 19,3 & - & - & \\
\hline Assistência pré-natal & 84 & 95,5 & 74 & 98,7 & 0,4656 \\
\hline
\end{tabular}


dante com o de outros autores brasileiros 5,15,23. $\mathrm{Na}$ literatura internacional os índices de preferência pelo parto vaginal reportados são ainda maiores: 93,5\% em estudo australiano, 96,3\% em estudo asiático e variando de 86 a 99,7\% em uma revisão sistemática de 17 artigos 24,25,26. Por outro lado, entre as mulheres da maternidade privada nesta pesquisa, a preferência pela cesariana foi referida por quase $70 \%$ das mesmas, o que está em desacordo com todos os estudos pesquisados e pode refletir uma maior assimilação da alegada "cultura de cesárea” entre elas.

O principal motivo para preferência pelo parto vaginal, em ambas as maternidades, foi o fato de a recuperação ser mais rápida, enquanto que pela cesariana, também em ambas, foi o medo da dor associada ao parto vaginal. Segundo Figueiredo et al. ${ }^{27}$, esse medo não é justificável, uma vez que o desconforto vivenciado pelas parturientes durante o trabalho de parto e no pósparto não é muito diferente entre as duas vias de parto. Ou seja, a dor do parto, mesmo sendo amenizada pelo uso de analgesia ou da realização da cesariana, faz-se sempre presente, desde o pré até o pós-parto, variando de intensidade conforme as condições físicas e emocionais da parturiente, assim como a qualidade da assistência a ela prestada nestes momentos.

Perpétuo et al. 23 verificaram que, entre as mulheres que já haviam realizado o parto pelas duas vias, a menor dor foi citada dez vezes mais freqüentemente como motivo para preferir a via vaginal. No entanto, grande parte das cesarianas realizadas na maternidade privada é eletiva, ou seja, com data e hora marcadas, sem haver trabalho de parto, sendo assim eliminado o componente álgico do pré-parto, o que poderia contribuir para a escolha desta via de parto.

Por outro lado, contrariando o senso comum, entre as mulheres que manifestaram preferência pela cesariana, em ambas as maternidades, $o$ medo de deformidade vaginal e do conseqüente prejuízo da atividade sexual como motivo para evitar o parto vaginal foi muito pouco referido pelas mesmas (5,9\% e 3,3\%, respectivamente), achado semelhante ao de Perpétuo et al. 23 .

Tomando-se por base a taxa de cesarianas preconizada pela OMS de até $15 \%$, os índices verificados neste estudo, de $46 \%$ e 97,8\%, respectivamente, na maternidade pública e na privada, podem ser considerados muito elevados. Essas taxas são também maiores que aquelas descritas para o Brasil, em torno de 35\% em geral e de $70 \%$ em serviços privados 1,28. Vale frisar que alguns especialistas médicos têm demonstrado que é possível manter taxas abaixo de $2 \%$ sem comprometer a qualidade da assistência e sem acarretar aumento do risco de morbimortalidade materna e perinatal, o que só realça a importância do problema em pauta 29,30.

Considerando a maternidade pública, o fato de a mesma ser uma instituição universitária, de referência para todo o estado, poderia justificar uma maior complexidade dos casos atendidos e, conseqüentemente, contribuir para o aumento do porcentual de cesarianas.

A assistência pré-natal tem sido apontada como um fator de redução do risco de complicações e da necessidade de cesariana 1,31,32. No presente trabalho, no entanto, a despeito de a quase totalidade das parturientes ter recebido tal assistência, considerada completa em 57\% dos casos da maternidade pública e $100 \%$ dos da privada, observaram-se altas taxas de cesarianas. Na maternidade privada, segundo Domingues et al. ${ }^{32}$, uma possível explicação seria a influência do médico, presumivelmente a favor da cesariana, contribuindo para esses resultados, aparentemente paradoxais.

O obstetra, para realizar uma assistência ao trabalho de parto, deve dispor de, no mínimo, 6 a 10 horas para acompanhamento, com exame clínico periódico de 2 em 2 horas, principalmente quando se trata de primíparas, nas quais a evolução do trabalho de parto costuma ser mais prolongada. Na assistência realizada no serviço privado, o obstetra que, por diversos motivos, não puder disponibilizar desse tempo para a assistência, acabará indicando a cesariana. Já no serviço público, a gestante, ao internar em trabalho de parto, terá à sua disposição, para exame clínico periódico, uma equipe de obstetras em regime de plantão, o que possibilita a continuidade do acompanhamento; assim, o motivo da realização das cesarianas estaria associado mais às reais indicações médicas (ameaça ao binômio materno-fetal) do que à "falta de tempo" e conveniência do obstetra.

De fato, corroborando essa suposição, verificou-se neste estudo, que na maternidade privada, além da preferência predominante pela cesariana manifestada pelas gestantes $(67,4 \%)$, dentre aquelas favoráveis ao parto vaginal (29 pacientes) apenas $2(7 \%)$ tiveram o seu desejo atendido, enquanto que $100 \%$ das que preferiam a cesariana acabaram realizando este tipo de parto.

Na maternidade pública, as taxas de concordância entre preferência da gestante e o tipo de parto realizado foram de 55,8\% para o parto vaginal e de apenas $38,2 \%$ para a cesariana, o que sugere que o desejo da mulher teve importância menor, prevalecendo as indicações clínicas para cada via de parto. Assim, a alta taxa de cesarianas verificada na maternidade pública poderia justificar-se mais por outros fatores, como o fato de o estudo restringir-se a primíparas e de es- 
ta instituição ser considerada de referência para atendimento de média e alta complexidades, como já citado.

Como frisam Fabri \& Murta 31, a pouca ou nenhuma orientação durante o pré-natal sobre as reais indicações de cesariana e os benefícios e desvantagens de cada tipo de parto, associada ao progresso científico e ao aprimoramento da técnica cirúrgica, caracterizam um modelo de assistência médica fortemente determinante da opção pelo parto operatório. Barbosa et al. 18 acrescem ainda que muitas vezes é transmitida à gestante, durante o pré-natal, a idéia infundada de que o parto cesáreo é mais seguro para a mãe e para o bebê e, portanto, uma forma mais "moderna" de parto.

Quase todas as cesarianas realizadas na maternidade pública tiveram uma indicação clínica registrada, sendo a desproporção céfalo-pélvica, a doença hipertensiva específica da gravidez (DHEG) e o sofrimento fetal as principais. $\mathrm{Na}$ maternidade privada havia documentação de indicação clínica em apenas pouco mais de $60 \%$ dos casos, sendo a desproporção céfalo-pélvica a mais referida, seguida do sofrimento fetal e da DHEG, ficando os demais casos rotulados como "a pedido".

Independentemente do tipo de parto realizado, o nível de satisfação das parturientes foi elevado nas duas populações (superior a 90\% na pública e de $100 \%$ na maternidade privada), devendo-se, no entanto, destacar que, pelo seu caráter subjetivo, a satisfação é um conceito difícil de interpretar neste contexto por estar muitas vezes imbricado com a qualidade da assistência médica recebida e com o resultado neonatal 4. Entretanto, a intenção de repetir a via de parto foi muito mais freqüente entre as mulheres que tiveram parto vaginal, também em ambos os serviços.

Analisando apenas a população da maternidade pública, em que houve um equilíbrio entre as vias de parto, verificou-se que o grupo da cesariana apresentava uma renda melhor e uma maior proporção de mulheres brancas. Além disso, observou-se uma tendência a uma maior proporção de cesarianas entre as mais escolarizadas, que pode não ter atingido a significância estatística devido ao pequeno número de mulheres com o 3 o grau nesta amostra.
Esses achados, aliados à alta taxa de cesarianas verificada entre as mulheres de melhor nível sócio-econômico atendidas na maternidade privada deste estudo, a exemplo de outros, vem corroborar o papel de fatores não-clínicos na escolha do parto cesáreo, relacionados a aspectos socioculturais da prática obstétrica, incluindo atitudes da equipe médica, da mulher e de sua família 11,12,23,33.

Também em nível populacional, num estudo que analisou 19 países da América Latina, Belizan et al. ${ }^{1}$ verificaram uma correlação positiva e significante entre a taxa de cesarianas e a renda per capita, a proporção de população urbana e o número de médicos por 10 mil habitantes. É instigante, pois, que mulheres socioeconomicamente menos favorecidas e, conseqüentemente, com maior risco de complicações no parto, tenham menor probabilidade de cesariana do que aquelas com baixo risco obstétrico e maior poder aquisitivo 12 .

Em síntese, como também comentam Freitas et al. 12 ao tratarem da "cultura da cesariana no Brasil”, o parto operatório passou a significar para a parturiente e sua família um atendimento médico diferenciado ("parto sem dor", "ausência de asfixia ao nascimento", "sexualidade preservada"), e para o obstetra, maior controle do ato médico e de seu horário de trabalho.

Os dados aqui apresentados, à semelhança dos citados por Santos et al. ${ }^{34}$ e Hotimsky et al. 35 são bastante relevantes, uma vez que o parto operatório não é isento de riscos, contribuindo para o aumento da morbimortalidade materna e fetal, além de acarretar elevação dos custos médico-hospitalares.

Altas taxas de cesarianas em primíparas, como demonstrado neste estudo, são particularmente preocupantes porque implicam alta probabilidade de futuras cesarianas, pois, na prática, uma cesariana prévia constitui indicação quase absoluta para nova cesariana, justificando-se o ditado "uma vez cesárea sempre cesárea".

Os resultados desta pesquisa, que abrangeu apenas dois serviços hospitalares do município, não podem evidentemente ser extrapolados para toda a população; no entanto, seus achados conferem importância ao tema e apontam claramente para a necessidade da realização de estudos de maior porte, que permitam um conhecimento mais amplo da nossa realidade. 


\section{Resumo}

Esta pesquisa objetivou analisar aspectos relacionados à escolha do tipo de parto em uma maternidade do serviço público e outra do serviço privado, em São Luís, Maranhão, Brasil. Trata-se de um estudo transversal abordando comparativamente 163 primíparas de uma maternidade pública e 89 de maternidade privada, com médias de idade de 21,63 \pm 5,24 e 28,8 $\pm 5,41$ anos, respectivamente. Preferiam o parto vaginal $79,1 \%$ das gestantes da maternidade pública, e a cesariana $67,4 \%$ das da maternidade privada $(p<0,0001)$. O parto cesáreo foi realizado em $46 \%$ das mulheres da maternidade pública e em 97,8\% das da maternidade privada $(p<0,0001)$. A satisfação das parturientes foi elevada com as duas vias de parto, porém o desejo de repetir foi mais freqüentemente referido por aquelas submetidas ao parto vaginal (71,6\% vs. 41,3\% na maternidade pública e $100 \%$ vs. 65,5\% na privada). Na maternidade pública, o subgrupo cesariana apresentava mais mulheres brancas e de melhor renda. A taxa de cesarianas foi, portanto, elevada nas duas maternidades, sendo significativamente maior no maternidade privada, observando-se ainda preferência pelo parto vaginal na pública e pela cesariana na maternidade privada.

Parto Obstétrico; Maternidades; Estudo Comparativo

\section{Referências}

1. Belizan JM, Althabe F, Barros FC, Alexander S. Rates and implications of cesarean sections in Latin America: ecological study. BMJ 1999; 319: 1397-400.

2. Scott JR. Putting elective cesarean into perspective. Obstet Gynecol 2002; 99:967-8.

3. Anderson GM. Making sense of rising caesarean section rates [Editorial]. BMJ 2004; 329:696-7.

4. Faisal-Cury A, Menezes PR. Fatores associados à preferência por cesariana. Rev Saúde Pública 2006; 40:226-32.

5. D’Orsi E, Chor D, Giffin K, Angulo-Tuesta A, Barbosa GP, Gama AS, et al. Factors associated with cesarean sections in a public hospital in Rio de Janeiro, Brazil. Cad Saúde Pública 2006; 22:2067-78.

6. Bergeron $V$. The ethics of cesarean section on maternal request: a feminist critique of the American College of Obstetricians and Gynecologists' position on patient-choice surgery. Bioethics 2007; 21:478-87.

7. Chigbu CO, Ezeome IV, Iloabachie GC. Cesarean section on request in a developing country. Int J Gynaecol Obstet 2007; 96:54-6.

\section{Colaboradores}

N. R. Mandarino participou da concepção do projeto, revisão bibliográfica, coleta de dados, tratamento estatístico e redação do artigo. M. B. C. Chein contribuiu com a revisão bibliográfica e redação do artigo. F. C. Monteiro Júnior participou da revisão bibliográfica, redação e revisão ortográfica do manuscrito. L. M. O. Brito participou da concepção do projeto, revisão crítica e aprovação final. Z. C. Lamy realizou a análise dos dados, revisão crítica, revisão bibliográfica e aprovação final. V. J. S. Nina participou da análise dos dados, revisão crítica, revisão estatística e aprovação final. E. G. Mochel contribuiu com a concepção do projeto, coleta de dados, revisão crítica e aprovação final. J. A. Figueiredo Neto participou da análise crítica e revisão final.
8. Lobel M, DeLuca RS. Psychosocial sequelae of cesarean delivery: review and analysis of their causes and implications. Soc Sci Med 2007; 64:2272-84.

9. Associação Nacional de Saúde Suplementar. Diretrizes e iniciativas em prol do parto normal. http://www.ans.gov.br/portalv4/site/noticias / noticia_24100.asp?secao=Home (acessado em 09/ Mar/2008).

10. Sociedade Civil Bem-Estar Familiar no Brasil. Pesquisa Nacional de Demografia e Saúde, 1996. Rio de Janeiro: Sociedade Civil Bem-Estar Familiar no Brasil; 1997.

11. Hopkins K. Are Brazilian women really choosing to delivery by cesarean? Soc Sci Med 2000; 51:725-40.

12. Freitas PF, Drachler ML, Leite JCC, Grassi PR. Desigualdade social nas taxas de cesariana em primíparas no Rio Grande do Sul. Rev Saúde Pública 2005; 39:761-7.

13. Faúndes A, Cecatti JG. A operação cesárea no Brasil: incidência, tendências, causas, conseqüências e propostas de ação. Cad Saúde Pública 1991; 7:150-73. 
14. Potter JE, Berquó E, Perpétuo IHO, Leal OF, Hopkins K, Souza MR, et al. Unwanted caesarean sections among public and private patients in Brazil: prospective study. BMJ 2001; 323:1555-8.

15. Habiba M, Kaminski M, Da Frè M, Marsal K, Bleker O, Librero J, et al. Caesarean section on request: a comparason of obstetricians' attitudes in eight European countries. Br J Obstet Gynaecol 2006; 113:647-56.

16. Leeman LM, Plante LA. Patient-choice vaginal delivery? Ann Fam Med 2006; 4:265-8.

17. Cotzias CS, Paterson-Brown S, Fisk NM. Obstetricians say yes to maternal request for elective cesarean section: a survey of current opinion. Eur J Obstet Gynecol Reprod Biol 2001; 97:15-6.

18. Barbosa GP, Giffin K, Angulo-Tuesta A, Gama AS, Chor D, D'Orsi E, et al. Parto cesáreo: quem o deseja? Em quais circunstâncias? Cad Saúde Pública 2003; 19:1611-20.

19. Penna L, Arulkumaran S. Cesarean section for non-medical reasons. Int J Gynaecol Obstet 2003; 82:399-409.

20. McFarlin BL. Elective cesarean birth: issues and ethics of an informed decision. J Midwifery Womens Health 2004; 49:421-9.

21. American College of Obstetricians and Gynecologists. ACOG Committee Opinion No. 395. Surgery and patient choice. Obstet Gynecol 2008; 111: 243-7.

22. Área Técnica de Saúde da Mulher, Departamento de Ações Programáticas Estratégicas, Secretaria de Atenção à Saúde, Ministério da Saúde. Pré-natal e puerpério: atenção qualificada e humanizada: manual técnico. 2a Ed. Brasília: Ministério da Saúde; 2006.

23. Perpétuo IHOP, Bessa GH, Fonseca MC. Parto cesáreo: uma análise da perspectiva das mulheres de Belo Horizonte. In: Anais do 11o Encontro Nacional de Estudos Populacionais. Caxambu: Associação Brasileira de Estudos Populacionais; 1998. p. 95-119.
24. Gamble JA, Creedy DK. Women's preference for a cesarean section: incidence and associated factors. Birth 2001; 28:101-10.

25. Chong ES, Mongelli M. Attitudes of Singapore women toward cesarean and vaginal deliveries. Int J Gynaecol Obstet 2003; 80:189-94.

26. McCourt C, Weaver J, Statham H, Beake S, Gamble J, Creedy DK. Elective cesarean section and decision making: a critical review of the literature. Birth 2007; 34:65-79.

27. Costa R, Figueiredo B, Pacheco A, Pais A. Tipo de parto: expectativas, experiências, dor e satisfação. Revista de Obstetrícia e Ginecologia 2003; 6: 256-306.

28. Instituto Brasileiro de Geografia e Estatística. Taxas de cesariana - 1999. http://www.ibge.gov.br (acessado em 12/Mai/2007).

29. Rockenschaub A. Technology free obstetrics at the Semmelweis Clinic. Lancet 1990; 335:977-88.

30. Durand AM. The safety of home birth: the farm study. Am J Public Health 1992; 82:450-3.

31. Fabri RH, Murta EFC. Tipos de parto e formas de assistência médica em Uberaba-MG. Rev Bras Ginecol Obstet 1999; 21:99-104.

32. Domingues RMSM, Santos EM, Leal MC. Aspectos da satisfação das mulheres com a assistência ao parto: contribuição para o debate. Cad Saúde Pública 2004; 20 Suppl 1:S52-62.

33. Carnel EF, Zanolli ML, Narcillo AM. Fatores de risco para indicação do parto cesáreo em Campinas (SP). Rev Bras Ginecol Obstet 2007; 29:34-40.

34. Santos LC, Amorim MMR, Porto AMF, Azevedo EB, Mesquita CC. Fatores prognósticos para o parto transvaginal em pacientes com cesárea anterior. Rev Bras Ginecol Obstet 1998; 20:342-9.

35. Hotimsky SN, Rattner D, Venancio SI, Bógus CM, Miranda MM. O parto como eu vejo... ou como eu o desejo? Expectativas de gestantes, usuárias do SUS, acerca do parto e da assistência obstétrica. Cad Saúde Pública 2002; 18:1303-11.

Recebido em 02/Out/2008

Versão final reapresentada em 17/Mar/2009

Aprovado em 06/Abr/2009 